\title{
Sustrato en soluciones nutritivas: cambios en propiedades físicas y químicas durante el desarrollo de Paphiopedilum insigne (Wall. Ex Lindl.) Pfitzer y Laelia autumnalis (Lex.) Lindl. (Orchidiaceae)
}

Substrate in nutrient solutions: changes in physical and chemical properties during Paphiopedilum insigne (Wall. Ex Lindl.) Pfitzer and Laelia autumnalis (Lex.) Lindl. (Orchidiaceae) development

\author{
Nadia Jiménez-Peña ${ }^{1^{*}}(\mathbb{D})$, Víctor Hugo Volke-Haller ${ }^{1}\left(\mathbb{D}\right.$, Martha E. Pedraza-Santos ${ }^{2}$
}

${ }^{1}$ Programa de Edafología, Colegio de Postgraduados, Campus Montecillo, Carretera México-Texcoco km 36.5, 56230, Montecillo, Texcoco, Estado de México, México. ${ }^{2}$ Facultad de Agrobiología, Universidad Michoacana de San Nicolás de Hidalgo, 60170, Uruapan Michoacán, México. *Autor para correspondencia: okavango_@hotmail.com

Fecha de recepción:

24 de junio de 2020

Fecha de aceptación:

20 de agosto de 2020

Publicado en línea:

4 de marzo de 2021

Este es un artículo en acceso abierto que se distribuye de acuerdo a los términos de la licencia Creative Commons.

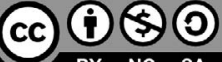

Reconocimiento-

NoComercial-

CompartirIgual 4.0

Internacional

\section{RESUMEN}

En el cultivo de orquídeas, un sustrato debe presentar un adecuado drenaje, aireación, humedad y alta estabilidad biológica, así como lenta degradación. El objetivo de este trabajo fue evaluar los cambios en las propiedades físicas y químicas de una mezcla de sustratos en las orquídeas Paphiopedilum insigne (Wall. Ex Lindl.) y Laelia autumnalis (Lex.) Lindl. (Orchidiaceae), en función de las soluciones hidropónicas aplicadas durante su desarrollo. El experimento consistió en la mezcla de cuatro sustratos: carbón, tezontle, corteza y turba-vermiculita en una relación 1:2:2:1, con dos especies de orquídeas y tres soluciones nutritivas: Wynd, Hoagland y Arnon y Steiner, y un testigo (agua de pozo). El experimento se llevó a cabo por un lapso de dos años y tres meses, en invernadero. Se encontró que las soluciones nutritivas afectaron las propiedades físicas y químicas del sustrato. Sin embargo, de las tres soluciones nutritivas y el testigo, la solución Hoagland y Arnon ocasionó menos cambios en las propiedades físicas y químicas del sustrato, así como poca retención de nutrimentos de la solución durante el periodo de evaluación, por lo cual es una buena alternativa para el desarrollo de estas dos orquídeas.

\section{PALABRAS CLAVE}

Epifitas, porosidad, macronutrimentos, micronutrimentos, cultivo sin suelo

\section{ABSTRACT}

In orchid cultivation, a substrate must present proper drainage, aeration, humidity and high biological stability, as well as slow degradation. The objective of this work was to assess the changes in the physical and chemical properties of a mixture of substrates in the Paphiopedilum insigne (Wall. Ex Lindl.) and Laelia autumnalis (Lex.) Lindl. (Orchidiaceae) orchids based on the hydroponic solutions applied during their development. The experiment consisted of the mixture of four substrates: coal, tezontle, bark and peat-vermiculite in a 1:2 2:1 ratio, with two species of orchids and three nutritional solutions: Wynd, Hoagland-Arnon and Steiner, and a control (well water). The experiment was carried out for a period of two years and three months, in a greenhouse. Nutrient solutions were found to affect the physical and chemical properties of the substrate. However, of the three nutrient solutions and the control, the Hoagland-Arnon solution caused fewer changes in the physical and chemical properties of the substrate, as well as little nutrient retention from the solution during the evaluation period, thus proving to be a good alternative for the development of these two orchids.

\section{KEYWORDS}

Epiphytes, porosity, macronutrients, micronutrients, soilless cultivation 


\section{INTRODUCCIÓN}

En los últimos años la producción comercial de orquídeas se ha expandido enormemente y se ha convertido en una industria rentable. Muchas especies de los géneros Cymbidium, Paphiopedilum y Phalaenopsis se cultivan por la belleza de sus flores (Hew y Yong 2004). El caso particular de las orquídeas actualmente representa una parte significativa del comercio mundial de flores, con ventas anuales de más de 4 mil millones de dólares en Estados Unidos (Zhang et al. 2018). Sin embargo, para la producción exitosa de plantas de alta calidad en macetas, se requiere de una comprensión del ambiente único encontrado en la maceta y de la manera en la que éste es afectado por las propiedades físicas y químicas de los sustratos utilizados (Cabrera 1995). Las propiedades físicas son consideradas las más importantes para un sustrato. Esto es debido a que, si la estructura física es inadecuada, difícilmente se podrá mejorar una vez que se ha establecido el cultivo. En cambio, las propiedades químicas sí pueden ser alteradas después del establecimiento del cultivo (Cabrera 1999).

En el cultivo de orquídeas epífitas en macetas, el sustrato influye en la calidad del producto final, sobre todo como soporte de las raíces. En su hábitat natural, estas plantas sólo utilizan al huésped para fijarse con sus raíces, las cuales quedan expuestas, gracias a lo cual pueden absorber el agua de la lluvia, el rocío y la humedad relativa de la noche, mientras que los nutrientes se derivan de la descomposición de materia orgánica depositada en el tronco. Por lo tanto, un sustrato para cultivar orquídeas en contenedores debe tener una textura ideal para el suministro de agua y aire a las raíces, con capacidad de retención alta de nutrimentos y $\mathrm{pH}$ adecuado, ya que las raíces pueden morir por falta o exceso de humedad; asimismo, la nutrición también debe ser equilibrada, de modo que no exista una reducción en el crecimiento y en la floración de las plantas (Demattê y Demattê 1996; Faria et al. 2001; De Assis et al. 2008).

$\mathrm{Al}$ respecto, se ha comprobado que las orquídeas reaccionan de manera distinta a los sustratos. En crecimiento y floración, Wang y Gregg (1994), en el caso de Phalaenopsis, sugieren sustratos con perlita, Metro Mix ${ }^{\circledR} 250$ y carbón vegetal en proporción 1:1:1, y perlita y lana de roca (1:1); Faria et al. (2001), en orquídeas nativas brasileñas Oncidium baueri Lindl. y Maxillaria picta Hook., encontraron que el mejor sustrato es vermiculita; en aclimatización, Arenas-de-Souza y Karsburg (2016), en Catasetum schmidtianum Miranda \& Lacerda, encontraron eficiente el sustrato musgo de Chile (Sphagnum magellanicum Brid) + vermiculita + paja carbonizada de arroz + carbón (1:1:1:1); para establecimiento en keikis de Epidendrum melinanthum Schltr., bajo condiciones ex situ, el uso de sustratos tanto nutritivos como inertes (pino patula, cachaza/carbonilla, cascarilla de arroz, arena, suelo), permitieron el establecimiento de las plántulas (Buitrón et al. 2016). Sin embargo, los autores no mencionan los cambios del sustrato o si existe descomposición a través del tiempo.

En adición a lo anterior, el uso de dosis frecuentes de soluciones nutritivas y fertilizantes influye en el crecimiento y floración (Jiménez-Peña et al. 2013; Jiménez-Peña et al. 2019; Tse y Khye 2007; Wang 2007). Sin embargo, existen escasos estudios en orquídeas que traten los cambios que sufren los sustratos en sus propiedades físicas y químicas, así como en la acumulación de nutrimentos con el paso del tiempo por efecto de los fertilizantes.

Por tal motivo, la presente investigación tuvo como objetivo evaluar los cambios en las propiedades físicas y químicas de una mezcla de sustratos en las orquídeas Paphiopedilum insigne (Wall. Ex Lindl.) y Laelia autumnalis (Lex.) Lindl., en función de las soluciones hidropónicas aplicadas durante su desarrollo.

\section{Materiales y Métodos}

\section{Material vegetal y sustrato}

Se usaron plantas de $P$. insigne, con una edad aproximada de un año y dos meses, y un tamaño medio de $15 \mathrm{~cm}$; asimismo, plantas adultas de L. autumnalis, de cinco a seis años, y un tamaño medio de $25 \mathrm{~cm}$. Las primeras se adquirieron con un proveedor comercial de Uruapan, Michoacán, México. Las segundas fueron proporcionadas por el banco de germoplasma del Sistema Nacional de Recursos Fitogenéticos (SINAREFI), originalmente colectadas en siete sitios de bosque de pino-encino 
de dos localidades de los municipios de Tzintzuntzan y Uruapan, Michoacán, México. Las plantas se trasplantaron (una planta por recipiente) en macetas oscuras de plástico de seis pulgadas de diámetro, con un volumen de $1.330 \mathrm{~L}$.

El sustrato evaluado consistió en una mezcla de carbón vegetal forestal (pino-encino) + tezontle + corteza de pino + turba-vermiculita en una relación de 1:2:2:1, con las características físicas de $64 \%$ de porosidad total, $26 \%$ de retención de agua y $38 \%$ de capacidad de aireación, y con un tamaño de partícula de 0.5 a $2 \mathrm{~cm}$ (Cuadro 1$)$.

Cuadro 1. Propiedades físicas y químicas iniciales del sustrato utilizado para el desarrollo de Paphiopedilum insigne y Laelia autumnalis (Orchidiaceae) en contenedor.

\begin{tabular}{cc}
\hline Variable & \\
\hline & Propiedades físicas \\
\cline { 2 - 2 } Porosidad total $(\%)$ & 64 \\
Porosidad de aireación $(\%)$ & 38 \\
Capacidad de retención de & 26 \\
agua $(\%)$ & 0.51 \\
Densidad aparente $\left(\mathrm{kg} / \mathrm{dm}^{3}\right)$ & 6.6 \\
& 2.0 \\
$\mathrm{pH}$ & 16.1 \\
$\mathrm{CE}\left(\mathrm{dS} \mathrm{m}^{-1}\right)$ & 1.4 \\
$\mathrm{CIC}$ & Propiedades químicas \\
\cline { 2 - 2 } $\mathrm{MO}(\%)$ & \\
\hline
\end{tabular}

Las plantas se colocaron en un invernadero de dos aguas, cubierto con plástico verde difuso y con malla sombra a $60 \%$ para evitar excesos de radiación. Asimismo, para evitar fuertes cambios de humedad y temperatura dentro del invernadero, se utilizó aspersión automatizada durante el día, con el objetivo de mantener fresco el ambiente. Durante los meses más fríos (noviembre-febrero) se utilizó calefacción por la noche para mantener la temperatura por arriba de los $11^{\circ} \mathrm{C}$.

Las plantas se irrigaron con tres soluciones nutritivas, Hoagland y Arnon (1938), Wynd (1933) y Steiner (1984) (Cuadro 2), utilizando sales comerciales y un testigo con agua de pozo, a la cual se le hizo un análisis químico para ajustar la concentración de los elementos de la solución nutritiva, además de adecuar el pH entre 5.5 y 6.0 con ácido sulfúrico.
Cuadro 2. Concentración (mg $\left.\mathrm{L}^{-1}\right)$ de nutrimentos en las soluciones nutritivas utilizadas para irrigar Paphiopedilum insigne y Laelia autumnalis (Orchidiaceae) en contenedor.

\begin{tabular}{cccc}
\hline Nutrimentos & $\begin{array}{c}\text { Hoagland } \\
\text { Arnon }\end{array}$ & Steiner & Wynd \\
\hline Nitrógeno & 210 & 167 & 225 \\
Fósforo & 31 & 31 & 137 \\
Potasio & 235 & 277 & 112 \\
Calcio & 160 & 183 & 169 \\
Magnesio & 49 & 49 & 25 \\
Azufre & 64 & 67 & 24 \\
Hierro & 5 & 3 & - \\
Manganeso & - & 1.97 & - \\
Boro & - & 0.44 & - \\
Zic & - & 0.11 & - \\
Cobre & - & 0.02 & - \\
Molibdeno & - & 0.007 & - \\
\hline
\end{tabular}

\section{Variables evaluadas}

Las variables evaluadas fueron propiedades físicas y químicas del sustrato después de desarrollar las plantas por dos años y tres meses. Las propiedades físicas fueron: porosidad total, porosidad de aireación, capacidad de retención de agua, densidad aparente determinadas mediante el uso de porómetros (Pire y Pereira 2003). Asimismo, se evaluaron las propiedades químicas: $\mathrm{pH}$ con un potenciómetro; conductividad eléctrica (CE), con un conductímetro; capacidad de intercambio catiónico (CIC), por el método de acetato de amonio; materia orgánica (MO), por calcinación; nitrógeno total $(\mathrm{Nt})$, por el método Semimicro-Kjeldahl; $\mathrm{P}, \mathrm{K}, \mathrm{Ca}, \mathrm{Mg}, \mathrm{S}, \mathrm{Fe}, \mathrm{Mn}, \mathrm{Cu}, \mathrm{Zn}, \mathrm{Cl}$ y Na, mediante el método de absorción atómica, el cual se da por medio de digestión húmeda del material seco con una mezcla de ácido perclórico-nítrico (Alcántar y Sandoval 1999).

\section{Diseño y análisis estadístico}

El diseño experimental fue completamente al azar, con la combinación de dos especies de orquídeas (L. autumnalis con ocho repeticiones y $P$. insigne con dieciséis repeticiones), con tres soluciones nutritivas (Hoagland y Arnon 1938, Steiner 1984 y Wynd 1933) y un testigo. La unidad experimental fue una planta con entre cinco y seis pseudobulbos (L. autumnalis), y una planta de tamaño pequeña de entre cuatro y cinco hojas en forma de cinta (P. insigne), en una maceta de 
plástico. Los datos obtenidos de los sustratos donde se desarrollaron las orquídeas se revisaron mediante análisis de varianza y comparación de medias de Tukey $(\mathrm{P} \leq 0.05)$ con el programa estadístico SAS para Windows v9.1 (SAS Institute 2003).

\section{RESUltados Y Discusión}

Para la elección del sustrato se hicieron varias mezclas, con el fin de obtener propiedades físicas adecuadas, como porcentaje de aireación mayor a $20 \%$ para el desarrollo de orquídeas epifitas - sugerido por Cabrera (1999) - , así como una lenta descomposición del sustrato (Cuadro 2). De acuerdo con Jiménez-Peña et al. (2013), sustratos formulados con carbón y turba en diferentes proporciones proveen las características físicas y químicas para un mejor desarrollo de hojas en orquídeas de Laelia anceps Lindl., aunque no existe un material que reúna todas las propiedades físicas y químicas óptimas para todos los usos, como señalan Segura et al. (2008). Las plantas de P. insigne y $L$. autumnalis respondieron favorablemente en su desarrollo al ser irrigadas por dos años tres meses con las distintas soluciones nutritivas; esto indica que, al término de la investigación, las propiedades de los sustratos de los tratamientos fueron las adecuadas para el cultivo de orquídeas.

\section{Propiedades físicas, químicas y nutrimentos del sustrato de cultivo de $P$. insigne}

El sustrato en en el que se desarrolló la orquídea $P$. insigne presentó diferencias estadísticamente significativas $(\mathrm{P} \leq 0.05)$ en todas las variables evaluadas. El sustrato irrigado con la solución Hoagland y Arnon presentó porcentajes de porosidad total y porosidad de aireación 10 y $26 \%$ superiores con respecto al testigo. En el caso de aireación, los resultados son ligeramente superiores a los niveles óptimos (mayores a $20 \%$ ) de sustratos para cultivo de orquídeas indicados por Ansorena (1994). La capacidad de retención de agua disminuyó $15.9 \%$ y la densidad aparente aumentó $18 \%$ con respecto al testigo (Cuadro 3); los intervalos obtenidos del sustrato en general se encuentran entre los valores bajos que van de 1.0 a $1.5 \mathrm{~g} \mathrm{~cm}-3$ para el cultivo de plantas en maceta (Cabrera 1999). A lo largo del cultivo, suele existir una degradación física del sustrato al desarrollo de las raíces que ocupan el espacio poroso, lo cual repercute en las propiedades físicas dentro del contenedor (López-Cuadrado et al. 2006).

El sustrato de cultivo de las plantas de $P$. insigne presentó diferencias significativas en todas las propiedades químicas evaluadas, con excepción del porcentaje de materia orgánica. El sustrato se acidificó, la CE se elevó y la CIC disminuyó por efecto de la solución nutritiva, en contraste con el testigo (Cuadro 3). De acuerdo con Wang y Konow (2002), los valores encontrados de $\mathrm{pH}$ y $\mathrm{CE}$ son considerados altos; sin embargo, esto aparentemente no tuvo efectos negativos en el crecimiento o desarrollo de las plantas. Los valores encontrados de $\mathrm{pH}$ y $\mathrm{CE}$ probablemente debieron su incremento a la gran cantidad de bicarbonatos $(5 \mathrm{meq}$ $\left.\mathrm{L}^{-1}\right)$ que, de manera natural, contiene el agua de pozo, la cual se utilizó para la preparación de las soluciones nutritivas, así como a la acumulación de sales de las soluciones nutritivas con el paso del tiempo.

En general, el sustrato presentó una disgregación física ligera, así como cambios en su estabilidad y propiedades, con el paso del tiempo, derivados de la actividad en el sustrato causado por la solución nutritiva. Sin embargo, el crecimiento de la planta y la actividad microbiana también presentaron cambios en el sustrato, sobre todo en los periodos de calentamiento en primavera y verano, los cuales dieron lugar a riegos más frecuentes y aumentaron la actividad (Wang y Konow 2002), lo cual, a su vez, modificó propiedades como granulometría, densidad, porosidad o distribución del agua y aire en el espacio poroso (López-Cuadrado et al. 2006).

Como se esperaba, el sustrato tratado con las distintas soluciones nutritivas presentó incrementos en $\mathrm{N}$ en un 50, P 295, K 222, Ca 152 y Mg 79\% con respecto al testigo (Cuadro 4). No obstante, estas concentraciones de macronutrimentos encontrados en el sustrato son bajos de acuerdo con Wang y Konow (2002). Sin embargo, Demattê y Graziano (2000) mencionan que concentraciones altas de $\mathrm{Ca}$ y $\mathrm{Mg}$ son perjudiciales para el crecimiento de plantas, y, con el fin de evitar la toxicidad, los componentes de sustratos para cultivos de orquídeas epifitas deben tener concentraciones relativamente bajas de nutrientes, como el caso Dendrobium nobile Lindl., que muestra una alta absorción. 
Cuadro 3. Efecto de la solución nutritiva sobre las propiedades físicas y químicas del sustrato utilizado para el desarrollo de Paphiopedilum insigne (Orchidiaceae) en contenedor.

\begin{tabular}{|c|c|c|c|c|}
\hline \multirow[b]{2}{*}{ Variable } & \multicolumn{4}{|c|}{ Solución nutritiva } \\
\hline & Agua & Wynd & Hoagland y Arnon & Steiner \\
\hline & \multicolumn{4}{|c|}{ Propiedades físicas } \\
\hline Porosidad total (\%) & $67.00^{1} \mathrm{~b}$ & $67.93 \mathrm{~b}$ & $73.41 \mathrm{a}$ & $63.45 \mathrm{~b}$ \\
\hline Porosidad de aireación (\%) & $36.98 \mathrm{~b}$ & $40.49 \mathrm{ab}$ & $46.54 \mathrm{a}$ & $39.89 \mathrm{ab}$ \\
\hline Capacidad de retención de agua (\%) & $28.89 \mathrm{a}$ & $29.92 \mathrm{a}$ & $28.38 \mathrm{ab}$ & $24.36 \mathrm{~b}$ \\
\hline \multirow[t]{2}{*}{ Densidad aparente $\left(\mathrm{kg} / \mathrm{dm}^{3}\right)$} & $0.40 \mathrm{~b}$ & $0.43 \mathrm{ab}$ & $0.46 \mathrm{ab}$ & $0.47 \mathrm{a}$ \\
\hline & \multicolumn{4}{|c|}{ Propiedades químicas } \\
\hline $\mathrm{pH}$ & $6.90 \mathrm{a}$ & $6.02 \mathrm{~b}$ & $6.48 \mathrm{bc}$ & $6.45 \mathrm{bc}$ \\
\hline $\mathrm{CE}\left(\mathrm{dS} \mathrm{m}^{-1}\right)$ & $2.17 \mathrm{~b}$ & $8.64 \mathrm{a}$ & $6.77 \mathrm{a}$ & $8.74 \mathrm{a}$ \\
\hline $\mathrm{CIC}$ & $18.37 \mathrm{a}$ & $15.02 \mathrm{ab}$ & $13.32 \mathrm{~b}$ & $12.64 \mathrm{~b}$ \\
\hline $\mathrm{MO}(\%)$ & $1.48 \mathrm{a}$ & $1.51 \mathrm{a}$ & $1.18 \mathrm{a}$ & $1.19 \mathrm{a}$ \\
\hline
\end{tabular}

${ }^{1}$ Para la comparación de medias, letras diferentes en una misma hilera indican medias distintas entre soluciones nutritivas.

Cuadro 4. Efecto de la solución nutritiva sobre macro y micronutrimentos del sustrato utilizado para el desarrollo Paphiopedilum insigne (Orchidiaceae) en contenedor.

\begin{tabular}{|c|c|c|c|c|}
\hline \multirow[b]{2}{*}{ Variable } & \multicolumn{4}{|c|}{ Solución nutritiva } \\
\hline & Agua & Wynd & Hoagland y Arnon & Steiner \\
\hline & \multicolumn{4}{|c|}{ Macronutrimentos $\left(\mathrm{mg} \mathrm{kg}^{-1}\right)$} \\
\hline Nitrógeno total (\%) & $0.04 \mathrm{~b}$ & $0.05 \mathrm{ab}$ & $0.06 \mathrm{ab}$ & $0.06 \mathrm{a}$ \\
\hline Fósforo & $11.60 \mathrm{bc}$ & $45.77 \mathrm{a}$ & $43.61 \mathrm{a}$ & $30.54 \mathrm{~b}$ \\
\hline Potasio & $0.64 \mathrm{~b}$ & $0.94 \mathrm{~b}$ & $1.04 \mathrm{~b}$ & $2.06 \mathrm{a}$ \\
\hline Calcio & $18.50 \mathrm{~b}$ & $28.03 \mathrm{a}$ & $23.63 \mathrm{ab}$ & 28.18 a \\
\hline Magnesio & $12.17 \mathrm{~b}$ & $21.78 \mathrm{a}$ & $16.08 \mathrm{ab}$ & $16.38 \mathrm{ab}$ \\
\hline \multirow[t]{2}{*}{ Azufre } & $180.88 \mathrm{bc}$ & $921.53 \mathrm{a}$ & $569.83 \mathrm{~b}$ & $619.72 \mathrm{~b}$ \\
\hline & \multicolumn{4}{|c|}{ Micronutrimentos $\left(\mathrm{mg} \mathrm{kg}^{-1}\right)$} \\
\hline Fierro & $502.25 \mathrm{a}$ & $478.38 \mathrm{ab}$ & $327.93 \mathrm{~b}$ & $356.75 \mathrm{ab}$ \\
\hline Manganeso & $78.39 a$ & $62.60 \mathrm{~b}$ & $67.71 \mathrm{ab}$ & $59.99 \mathrm{~b}$ \\
\hline Zinc & $6.73 \mathrm{~b}$ & $7.04 \mathrm{~b}$ & $11.37 \mathrm{a}$ & $10.87 \mathrm{a}$ \\
\hline Cobre & $4.08 \mathrm{~b}$ & $8.32 \mathrm{ab}$ & $14.98 \mathrm{a}$ & $13.50 \mathrm{a}$ \\
\hline \multirow[t]{2}{*}{ Cloro } & $3.37 \mathrm{~b}$ & $4.06 \mathrm{ab}$ & $4.38 \mathrm{a}$ & $4.67 \mathrm{a}$ \\
\hline & \multicolumn{4}{|c|}{ Elemento benéfico (mg kg-1) } \\
\hline Sodio & $0.75 \mathrm{~b}$ & $9.35 \mathrm{a}$ & $8.35 \mathrm{a}$ & $0.77 \mathrm{~b}$ \\
\hline
\end{tabular}

${ }^{1}$ Para la comparación de medias, letras diferentes en una misma hilera indican medias distintas entre soluciones nutritivas.

La retención de iones de los macronutrimentos es causada por diferentes factores, como son: tipo y cantidad de arcillas presentes, cantidad de materia orgánica y $\mathrm{pH}$. A medida que se va utilizando un sustrato, se acumula $\mathrm{MO}$, resultante de los restos de raíces y degradación del sustrato; los efectos de esta acumulación se manifiestan sobre la solución nutritiva con la formación de complejos órgano-minerales insolubles con los iones $\mathrm{Fe}, \mathrm{Al}$ y fosfatos, principalmente. Estos complejos pueden causar estados deficitarios a las plantas; posteriormente, al disociarse estos complejos por descomposición de la $\mathrm{MO}$, los iones vuelven a quedar en libertad, lo que puede producir efectos tóxicos (Landis 1989).

Para el caso de micronutrimentos también existió un incremento mayor en el sustrato, por efecto de 
las soluciones nutritivas en $\mathrm{Zn}$ hasta en 69, $\mathrm{Cu} 267$, Cl 39\% y el elemento benéfico $\mathrm{Na} 427 \%$ con respecto al testigo, con las excepciones de Fe y Mn (Cuadro 4). Sin embargo, Fe y Mn presentan concentraciones altas que en un futuro pueden ocasionar problemas en el desarrollo de la planta. Wang y Konow (2002) mencionan concentraciones que van de 0.02-0.76 en Fe y 2.9-4.7 $\mathrm{mg} \mathrm{L}^{-1}$ en $\mathrm{Mn}$; sin embargo, en el estudio, las plantas no tuvieron disponibilidad de micronutrimentos en el fertirriego, los cuales pudieran ser mayores. En la interacción Fe x Mn, cantidades demasiado elevadas de $\mathrm{Mn}$ en el medio en relación con Fe puede inducir síntomas de clorosis de Fe. El antagonismo no es a nivel de absorción, sino al de la actividad enzimática; el Mn compite en las locaciones metabólicas ocupadas normalmente por el Fe (Loué 1988).

Las concentraciones de $\mathrm{Cl}$ y $\mathrm{Na}$ fueron bajas; estos iones tienen un efecto negativo y pueden causar toxicidad cuando su concentración es excesiva (Villa et al. 2006). El $\mathrm{Na}$ afecta la estructura del sustrato, ocasionando que pierda las propiedades adecuadas para el cultivo; esto provoca, sobre todo, una disminución en la aireación y, por lo tanto, una disminución en la entrada de agua en el sustrato (infiltración).

\section{Propiedades físicas, químicas y macronutrimentos del sustrato de cultivo de L. autumnalis}

En el caso del sustrato donde se cultivó la orquídea $L$. autumnalis, la tendencia fue diferente a $P$. insigne. Las propiedades físicas (capacidad de retención de agua y densidad aparente) y químicas ( $\mathrm{pH}, \mathrm{CE}, \mathrm{CIC}$ y MO) no tuvieron incrementos en los tratamientos con respecto al testigo; en tanto que porosidad total y porosidad de aireación sí registraron diferencias (Cuadro 5). Las propiedades físicas aún presentan los requisitos mínimos para la producción de plantas en maceta; sin embargo, el $\mathrm{pH}$ y la $\mathrm{CE}$ presentaron valores altos, por lo que pudieran ocasionar el bloqueo de algunos nutrimentos para las plantas, así como la formación de carbonatos de calcio y magnesio (Steiner 1984). Ambos efectos son atribuidos a la falta de lavado de sales del sustrato - como se recomienda para evitar la salinización y la reducción en el rendimiento de la planta
(Goykovic y Saavedra 2007) - y a la gran cantidad de bicarbonatos que contiene de manera natural el agua de pozo, como se mencionó anteriormente.

Las soluciones nutritivas nuevamente ocasionaron incrementos en macronutrimentos con respecto al testigo. Se incrementó N en un 50, P 167, Ca 34 y Mg $167 \%$. Elementos como Ca y $\mathrm{Mg}$ no son fácilmente lixiviables, por lo que quedan disponibles para la planta en periodos largos (Farnham et al. 1985). Sin embargo, un exceso de sales fertilizantes puede restringir severamente el crecimiento de las plantas (por inducción de estrés hídrico específico de ciertos iones), lo que ocasiona daños indeseables al follaje e inclusive la muerte de las plantas, como menciona Bunt (1988). En el caso de los micronutrimentos, no hubo incremento de éstos por efecto de las soluciones nutritivas con respeto al testigo (Cuadro 6). En este sentido, la fuente de las soluciones nutritivas proporcionó la demanda de plantas en crecimiento y desarrollo respecto a las plantas testigo, las cuales fueron de menor tamaño, por lo que no demandaron los micronutrimentos y éstos permanecieron en el sustrato (Taiz et al. 2018).

\section{Conclusiones}

La solución nutritiva Hoagland y Arnon ocasionó los menores cambios en las propiedades físicas y químicas del sustrato durante un periodo de dos años y tres meses, por lo cual la mezcla de sustratos: carbón, tezontle, corteza y turba-vermiculita en una relación de 1:2:2:1 es una mezcla adecuada para el desarrollo de las orquídeas Paphiopedilum insigne y Laelia autumnalis desarrolladas en maceta y en condiciones hidropónicas. 
Cuadro 5. Efecto de la solución nutritiva sobre las propiedades físicas y químicas del sustrato utilizado para el desarrollo Laelia autumnalis (Orchidiaceae) en contenedor.

\begin{tabular}{|c|c|c|c|c|}
\hline \multirow[b]{2}{*}{ Variable } & \multicolumn{4}{|c|}{ Solución nutritiva } \\
\hline & Agua & Wynd & Hoagland y Arnon & Steiner \\
\hline & \multicolumn{4}{|c|}{ Propiedades físicas } \\
\hline Porosidad total $(\%)$ & $73.92^{1} \mathrm{a}$ & $64.54 \mathrm{~b}$ & $62.49 \mathrm{~b}$ & $60.84 \mathrm{~b}$ \\
\hline Porosidad de aireación (\%) & $50.28 \mathrm{a}$ & $38.89 \mathrm{~b}$ & $40.16 \mathrm{~b}$ & $38.60 \mathrm{~b}$ \\
\hline Capacidad de retención de agua (\%) & $23.63 \mathrm{a}$ & $25.66 \mathrm{a}$ & $22.33 \mathrm{a}$ & $22.24 \mathrm{a}$ \\
\hline \multirow{2}{*}{ Densidad aparente $\left(\mathrm{kg} / \mathrm{dm}^{3}\right)$} & $0.42 \mathrm{a}$ & $0.41 \mathrm{a}$ & $0.37 \mathrm{a}$ & $0.40 \mathrm{a}$ \\
\hline & \multicolumn{4}{|c|}{ Propiedades químicas } \\
\hline $\mathrm{pH}$ & $6.78 \mathrm{a}$ & $6.20 \mathrm{~b}$ & $6.20 \mathrm{~b}$ & $6.52 \mathrm{ab}$ \\
\hline $\mathrm{CE}(\mathrm{dS} / \mathrm{m})$ & 8.98 a & $8.07 \mathrm{a}$ & $7.30 \mathrm{a}$ & $6.73 \mathrm{a}$ \\
\hline CIC & $13.44 \mathrm{a}$ & $10.64 \mathrm{ab}$ & $10.92 \mathrm{ab}$ & $9.50 \mathrm{~b}$ \\
\hline $\mathrm{MO}(\%)$ & $1.66 \mathrm{a}$ & $1.61 \mathrm{a}$ & $1.66 \mathrm{a}$ & $1.32 \mathrm{a}$ \\
\hline
\end{tabular}

${ }^{1}$ Para la comparación de medias, letras diferentes en una misma hilera indican medias distintas entre soluciones nutritivas

Cuadro 6. Efecto de la solución nutritiva sobre macro y micronutrimentos del sustrato utilizado para el desarrollo Laelia autumnalis (Orchidiaceae) en contenedor.

\begin{tabular}{|c|c|c|c|c|}
\hline \multirow[b]{2}{*}{ Variable } & \multicolumn{4}{|c|}{ Solución nutritiva } \\
\hline & Agua & Wynd & Hoagland y Arnon & Steiner \\
\hline & \multicolumn{4}{|c|}{ Macronutrimentos $\left(\mathrm{mg} \mathrm{kg}^{-1}\right)$} \\
\hline Nitrógeno total (\%) & $0.04 \mathrm{~b}$ & $0.05 \mathrm{a}$ & $0.06 a$ & $0.06 a$ \\
\hline Fósforo & $12.62 \mathrm{bc}$ & $32.67 \mathrm{a}$ & $33.69 a$ & $23.46 b$ \\
\hline Potasio & $0.64 \mathrm{~b}$ & $0.56 \mathrm{~b}$ & $0.75 b$ & $1.65 a$ \\
\hline Calcio & $21.33 \mathrm{~b}$ & $23.13 \mathrm{ab}$ & $28.50 \mathrm{a}$ & $27.00 \mathrm{a}$ \\
\hline Magnesio & $13.83 \mathrm{~b}$ & $23.20 \mathrm{a}$ & $16.00 \mathrm{~b}$ & $13.80 \mathrm{~b}$ \\
\hline \multirow[t]{2}{*}{ Azufre } & $257.85 \mathrm{~b}$ & $965.39 \mathrm{a}$ & $613.49 \mathrm{ab}$ & $474.28 b$ \\
\hline & \multicolumn{4}{|c|}{ Micronutrimentos $\left(\mathrm{mg} \mathrm{kg}^{-1}\right)$} \\
\hline Fierro & $567.85 \mathrm{a}$ & $380.05 \mathrm{bc}$ & $453.66 \mathrm{~b}$ & $346.88 \mathrm{bc}$ \\
\hline Manganeso & $72.68 \mathrm{a}$ & $50.32 \mathrm{bc}$ & $63.52 \mathrm{~b}$ & $57.45 \mathrm{~b}$ \\
\hline Zinc & 12.38 a & $9.68 \mathrm{ab}$ & $8.78 \mathrm{ab}$ & $6.17 \mathrm{~b}$ \\
\hline Cobre & $7.74 \mathrm{ab}$ & $10.60 \mathrm{ab}$ & $12.80 \mathrm{a}$ & $6.00 \mathrm{~b}$ \\
\hline \multirow[t]{2}{*}{ Cloro } & $4.92 \mathrm{a}$ & $3.16 \mathrm{~b}$ & $3.59 \mathrm{~b}$ & $3.85 \mathrm{~b}$ \\
\hline & \multicolumn{4}{|c|}{ Elemento benéfico (mg kg-1) } \\
\hline Sodio & $0.78 \mathrm{~b}$ & $4.22 \mathrm{ab}$ & $6.81 \mathrm{a}$ & $7.18 \mathrm{a}$ \\
\hline
\end{tabular}

${ }^{1}$ Para la comparación de medias, letras diferentes en una misma hilera indican medias distintas entre soluciones nutritivas. 


\section{LITERATURA CITADA}

Alcántar GG, Sandoval VM. 1999. Manual de análisis químico de tejido vegetal. Guía de muestreo, preparación, análisis e interpretación. Publicación especial núm. 10. Sociedad Mexicana de la Ciencia del Suelo, A. C. Chapingo, México.

Ansorena MJ. 1994. Sustratos propiedades y caracterización. Mundi-Prensa. Madrid, España.

Arenas-de-Souza M, Karsburg IV. 2016. Substratos alternativos na aclimatação de Catasetum schmidtianum Miranda e Lacerda (Orchidaceae) micropropagadas. Revista Biociências 22: 36-41.

Buitrón MI, Pinta AE, Otero JT, Bonilla MM. 2016. Evaluación de sustratos para el establecimiento de keikis de Epidendrum melinanthum Schltr. (Orchidaceae: Laelinae) bajo condiciones de invernadero. Revista Facultad de Ciencias Básicas 12: 136-141. https://doi. org/10.18359/rfcb.2024

Bunt AC. 1988. Media and Mixes for Container-Grown Plants. Unwin Hyman Ltd. London, UK.

Cabrera RI. 1995. Fundamentals of container media management, Part. 1. Physical properties. Cooperative Extension Leaflet No. 950. University of California. New Jersey, USA.

Cabrera RI. 1999. Propiedades, uso y manejo de sustratos de cultivo para la producción de plantas en maceta. Revista Chapingo Serie Horticultura 5: 5-11. https://doi. org/10.5154/r.rchsh.1998.03.025

De Assis AM, de Faria RT, Unemoto LL, Colombo LA. 2008. Cultivo de Oncidium baueri Lindley (Orchidaceae) em substratos a base de coco. Ciência e Agrotecnologia 3: 981985. https://doi.org/10.1590/S1413-70542008000300042

Demattê JBJ, Demattê MESP. 1996. Estudos hídricos com substratos vegetais para cultivo de orquídeas epífitas. Pesquisa Agropecuária Brasileira 11: 803-813.

Demattê MESP, Graziano TT. 2000. Growth of Dendrobium nobile Lindl. as related with nutrient concentration in the growing media. Acta Horticulturae 511: 265-270. https:// doi.org/10.17660/ActaHortic.2000.511.30

de Faria RT, Rego LV, Bernardi HB, Molinari HB. 2001. Performance of different genotyps of Brazilian orchid cultivation in alternatives substrates. Brazilian Archives of Biology and Technology 44: 337-342. https://doi. org/10.1590/S1516-89132001000400003

Farnham DS, Hasek RF, Paul JL. 1985. Water quality; its effects on ornamental plants. Cooperative Extension
Leaflet No. 2995. University of California. New Jersey, USA.

Goykovic CV, Saavedra RG. 2007. Algunos efectos de la salinidad en el cultivo del tomate y prácticas agronómicas de su manejo. IDESIA (Chile) 25: 47-58. https://doi. org/10.4067/S0718-34292007000300006

Hew CS, Yong JW. 2004. The Physiology of Tropical Orchids in Relation to the Industry. World Scientific. Jurong East, Singapore.

Hoagland DR, Arnon DI. 1938. The water-culture method for growing plants without soil. California Agricultural Experiment Station Circular 347. University of California. Berkeley, USA.

Jiménez-Peña N, Valdez-Aguilar LA, Castillo-González AM, Colinas-León MT, Cartmill AD, Cartmill DL. 2013. Growing media and nutrient solution concentration affect vegetative growth and nutrition of Laelia anceps Lindl. HortScience 48: 773-779. https://doi.org/10.21273/ HORTSCI.48.6.773

Jiménez-Peña N, Sandoval-Villa M, Volke-Haller V, Pedraza-Santos M, Colinas-León MT. 2019. La solución nutritiva modifica el crecimiento de dos especies de orquídeas. Revista Fitotecnia Mexicana 42: 419-427. https://doi.org/10.35196/rfm.2019.4.419-427

Landis TD. 1989. Mineral nutrients and fertilization. In: Landis TD, Tinus RW, McDonald SE, Barnett JP, editors. The Container Tree Nursery Manual, vol. 4: Seedling Nutrition and Irrigation. Washington, DC, United States Department of Agriculture/Forest Service. P. 1-67.

López-Cuadrado MC, Ruiz-Fernández J, Masaguer A. 2006. Producción de planta ornamental en contenedor con sustratos alternativos a la turba: ensayos en la Comunidad de Madrid. Instituto Madrileño de Investigación y Desarrollo Rural, Agrario y Alimentario. Madrid, España.

Loué A. 1988. Los microelementos en la agricultura. MundiPrensa. Madrid, España.

Pire R, Pereira A. 2003. Propiedades físicas de componentes de sustratos de uso común en la horticultura del Estado Lara, Venezuela. Propuesta metodológica. Bioagro 15: 55-63.

[SAS] Statistical Analysis System. 2003. SAS/STAT User's Guide Release 9.1. SAS Institute. Cary, USA.

Segura CMA, Preciado RP, González CG, Frías RJE, García LG, Orozco VJA, Enríquez SM. 2008. Adición de material pomáceo a sustratos de arena para incrementar la capacidad de retención de humedad. Interciencia 12: 923-928. 
Steiner AA. 1984. The Universal Nutrition Solutions. Proceeding Sixth International Congress on Soilless Culture, ISOSC. Lunteren, The Netherlands.

Taiz L. Zeiger E. Møller IM. Murphy A. 2018. Fundamentals of Plant Physiology. Oxford University Press.

Tse LAC, Khye TTK. 2007. Versatile hydroponic technology for commercial orchid cultivation. Acta Horticulturae 742: 75-83. https://doi.org/10.17660/ ActaHortic.2007.742.10

Villa M, Catalán EA, Inzunza MA, Ulery AL. 2006. Absorción y traslocación de sodio y cloro en plantas de chile fertilizadas con nitrógeno y crecidas con estrés salino. Revista Fitotecnia Mexicana 29: 79-88.

Wang Y-T. 2007. Potassium nutrition affects Phalaenopsis growth and flowering. HortScience 42: 1563-1567. https://doi.org/10.21273/HORTSCI.42.7.1563

Wang Y-T, Gregg LL. 1994. Medium and fertilizer affect the performance of Phalaenopsis orchids during two flowering cycles. Hortscience 29: 269-271. https://doi. org/10.21273/HORTSCI.29.4.269

Wang Y-T, Konow EA. 2002. Fertilizer source and medium composition affect vegetative growth and mineral nutrition of a hybrid moth orchid. Journal of the American Society for Horticultural Science 127: 442-447. https:// doi.org/10.21273/JASHS.127.3.442

Wynd FL. 1933. Sources of carbohydrate for germination and growth of orchid seedlings. Annals of the Missouri Botanical Garden 20: 569-581. https://doi. org/10.2307/2394196

Zhang S, Yang Y, Li J, Qin J, Zhang W, Huang W, Hu H. 2018. Physiological diversity of orchids. Plant Diversity 40: 196-208. https://doi.org/10.1016/j.pld.2018.06.003 